\title{
Supporting Information: Impact of the Condensed-Phase Environment on the Translation-Rotation Eigenstates and Spectra of a Hydrogen Molecule in Clathrate Hydrates
}

\author{
Anna Powers, ${ }^{1}$ Ondrej Marsalek, ${ }^{1}$ Minzhong Xu, ${ }^{1}$ Lorenzo Ulivi, ${ }^{2}$ \\ Daniele Colognesi, ${ }^{2}$ Mark E. Tuckerman*, 3,4 and Zlatko Bačić ${ }^{1,4}$ \\ ${ }^{1}$ Department of Chemistry, New York University, New York, NY 10003, USA \\ ${ }^{2}$ Consiglio Nazionale delle Ricerche, Istituto dei Sistemi Complessi, \\ Via Madonna del Piano 10, I-50019, Sesto Fiorentino, Italy \\ ${ }^{3}$ Department of Chemistry and Courant Institute of Mathematical Sciences, \\ New York University, New York, NY 10003, USA \\ ${ }^{4}$ NYU-ECNU Center for Computational Chemistry at NYU Shanghai, \\ 3663 Zhongshan Road North, Shanghai, 200062, China
}

\section{S1. QUANTUM 5D CALCULATION OF THE TRANSLATION-ROTATION EIGENSTATES}

Our 5D bound-state approach ${ }^{1-3}$ utilizes the set of five coordinates $(x, y, z, \theta, \phi) ; x, y$, and $z$ are the Cartesian coordinates of the c.m. of $\mathrm{H}_{2}$, while the two polar angles $\theta$ and $\phi$ specify its orientation relative to the cage. The clathrate domains in Figure 1 are assumed to be rigid, infinitely heavy, and nonrotating. Then, the 5D TR Hamiltonian of the caged $\mathrm{H}_{2}$ is ${ }^{1}$

$$
H=-\frac{\hbar^{2}}{2 m}\left(\frac{\partial^{2}}{\partial x^{2}}+\frac{\partial^{2}}{\partial y^{2}}+\frac{\partial^{2}}{\partial z^{2}}\right)+B \mathbf{j}^{2}+V_{\mathrm{H}_{2}-\text { domain }}(x, y, z, \theta, \phi) .
$$

In eq $\mathrm{S} 1, m$ is the mass of the $\mathrm{H}_{2}$ molecule, $\mathbf{j}^{2}$ is the angular momentum operator of the diatomic, while $B$ denotes the rotational constant of the endohedral $\mathrm{H}_{2}\left(B=59.322 \mathrm{~cm}^{-1}\right.$ in this work $) . V_{\mathrm{H}_{2} \text {-domain }}(x, y, z, \theta, \phi)$ in eq $\mathrm{S} 1$ is the 5D intermolecular PES for the interaction between the entrapped $\mathrm{H}_{2}$ and the water molecules within the clathrate domain considered, which is described below. The TR eigenstates of the Hamiltonian in eq S1 are obtained utilizing the computational methodology that originated in our group, ${ }^{1-3}$ and is described in detail in ref 3 . The dimension of the sinc-discrete variable representation (DVR) basis was 19 for each of the three Cartesian coordinates, spanning the range -1.90 a.u. $\leq \lambda \leq 1.90$ a.u. $(\lambda=x, y, z)$. The angular basis included rotational functions up to $j_{\max }=5$. The energy cutoff parameter for the intermediate 3D eigenvector basis ${ }^{4}$ was set to $1600 \mathrm{~cm}^{-1}$.

\section{S2. 5D INTERMOLECULAR $\mathrm{H}_{2}$-CLATHRATE HYDRATE POTENTIAL ENERGY SURFACE}

The 5D intermolecular PES for the interaction between the entrapped $\mathrm{H}_{2}$ and the water molecules within the clathrate domain considered, denoted as $V_{\mathrm{H}_{2} \text {-domain }}$, is assumed to be pairwise additive. ${ }^{1,2,5-10}$ Hence, for $N \mathrm{H}_{2} \mathrm{O}$ molecules within the domain (with $N$ ranging from 20 to 1872), $V_{\mathrm{H}_{2}-\text { domain }}$ can be written as

$$
V_{\mathrm{H}_{2}-\text { domain }}\left(\mathbf{q}_{h}\right)=\sum_{w=1}^{N} V_{\mathrm{H}_{2}-\mathrm{H}_{2} \mathrm{O}}\left(\mathbf{q}_{h}, \boldsymbol{\Xi}_{w}\right),
$$

where $\mathbf{q}_{h}$ are the coordinates $(x, y, z, \theta, \phi)$ of $\mathrm{H}_{2}, V_{\mathrm{H}_{2}-\mathrm{H}_{2} \mathrm{O}}$ is the pair interaction between $\mathrm{H}_{2}$ and a framework $\mathrm{H}_{2} \mathrm{O}$ molecule, and the index $w$ runs over the water molecules of the domain, whose coordinates $\boldsymbol{\Xi}_{w}$ are fixed. $V_{\mathrm{H}_{2}-\text { domain }}$ is based on the interaction potential by Alavi et al., ${ }^{11}$ where the pair interaction $V_{\mathrm{H}_{2}-\mathrm{H}_{2} \mathrm{O}}$ in eq $\mathrm{S} 2$, is given by

$$
V_{\mathrm{H}_{2}-\mathrm{H}_{2} \mathrm{O}}=\sum_{i\left(\mathrm{H}_{2}\right)} \sum_{j\left(\mathrm{H}_{2} \mathrm{O}\right)} \frac{q_{i} q_{j}}{r_{i j}}+4 \epsilon_{\mathrm{O}-\mathrm{H}_{2}}\left[\left(\frac{\sigma_{\mathrm{O}-\mathrm{H}_{2}}}{r_{\mathrm{O}-\mathrm{H}_{2}}}\right)^{12}-\left(\frac{\sigma_{\mathrm{O}-\mathrm{H}_{2}}}{r_{\mathrm{O}-\mathrm{H}_{2}}}\right)^{6}\right] \text {. }
$$

The charges $j=1-3$ on the $\mathrm{H}_{2} \mathrm{O}$ molecule are taken from the SPC/E effective pair potential model for water, ${ }^{12}$ $-0.8476 e$ on the $\mathrm{O}$ atom and $+0.4238 e$ on each $\mathrm{H}$ atom. The point charges $i=1-3$ are placed on the $\mathrm{H}_{2}$ molecule, two

\footnotetext{
* Electronic mail: mark.tuckerman@nyu.edu

† Electronic mail: zlatko.bacic@nyu.edu
} 
positive charges of $+0.4932 e$ on its $\mathrm{H}$ atoms and one negative charge of $-0.9864 e$ at the c.m. of $\mathrm{H}_{2}$. They reproduce the experimental gas-phase quadrupole moment of $\mathrm{H}_{2}$. The second term in eq S3 represents the Lennard-Jones (LJ) interaction between the $\mathrm{O}$ atom of $\mathrm{H}_{2} \mathrm{O}$ and the c.m. of $\mathrm{H}_{2}$. The LJ parameters $\epsilon_{\mathrm{O}-\mathrm{H}_{2}}$ and $\sigma_{\mathrm{O}-\mathrm{H}_{2}}$ are determined following the standard Lorentz-Berthelot combination rules, and their values are given in Table II of ref $11 ; r_{\mathrm{O}-\mathrm{H}_{2}}$ is the distance between the $\mathrm{O}$ atom of $\mathrm{H}_{2} \mathrm{O}$ and the c.m. of $\mathrm{H}_{2}$.

$V_{\mathrm{H}_{2} \text {-domain }}$ in eq $\mathrm{S} 2$ is evaluated by direct summation of the LJ and electrostatic terms. To check that the long-range electrostatic interactions are converged, test calculations were also performed with the potential energy evaluated in the periodic boundary conditions utilizing the PME method ${ }^{13,14}$ with a $13 \AA$ cutoff for the short-range part of the electrostatic interaction. Lennard-Jones interaction were smoothly switched off between 11.5 and $12 \AA$. The GROMACS program ${ }^{15}$ was used to evaluate the interactions in the periodic boundary conditions.

\section{S3. RESULTS}

Figure 2 reveals that the first three solvation shells inside the radius $r=9.0 \AA$, containing 72 water molecules, account for most of the condensed-phase effects on the $j=1$ rotational splitting of the $\mathrm{H}_{2}$ molecule inside the central small cage. The following analysis aims to shed additional light on this finding. As discussed in the main text, the angular anisotropy of the $\mathrm{H}_{2}$-host interaction responsible for the $j=1$ splitting arises primarily from the long-range Coulomb interactions between the $\mathrm{H}_{2}$ quadrupole moment and the dipole moments of the framework water molecules. In Figure S1, the 2D electrostatic potential for $\mathrm{H}_{2}$ with its c.m. fixed at the center of the confining small cage is decomposed into the separate contributions from each of the first three solvation shells and the condensed-phase environment beyond them, as defined in the figure caption and the main text. The panels in the left column show the contributions from the individual shells, while those in the right column display the cumulative contributions from all water molecules within a given distance from $\mathrm{H}_{2}$ in the small cage. They make it clear that the main contributions to the electrostatic potential and its anisotropy come from the first three solvation shells within $9.0 \AA$ from $\mathrm{H}_{2}$, while the water molecules at larger distances contribute much less. In particular, the cumulative electrostatic potential for $r=0.00-9.00 \AA$ in the right column, due to 72 water molecules, differs little from that for $r=0.00-25.65$ $\AA$ from 1872 water molecules representing the condensed phase. This is reflected in the similar values of the $j=1$ rotational splittings calculated for these two domains, 32.18 and $31.66 \mathrm{~cm}^{-1}$, respectively. It should be pointed out that although the radial dependence of the electrostatic potentials displayed in Figure S1 obtained for one particular small cage (out of 432) can be considered typical, representative of all others, the quantitative variations among the 432 distinct small cages are substantial.

The effects of the proton disorder on the $j=1$ rotational level of the entrapped $\mathrm{H}_{2}$ are displayed in Fig. S2, where the distribution of its three components calculated for 432 small cages in their respective condensed-phase environments of $1872 \mathrm{H}_{2} \mathrm{O}$ molecules is shown. Despite the significant broadening due to the proton disorder, this distribution still exhibits three well separated peaks, consistent with the triplet structure of the rotational $j=0 \rightarrow 1$ transition clearly visible in the experimental INS spectrum. ${ }^{6,8}$ 


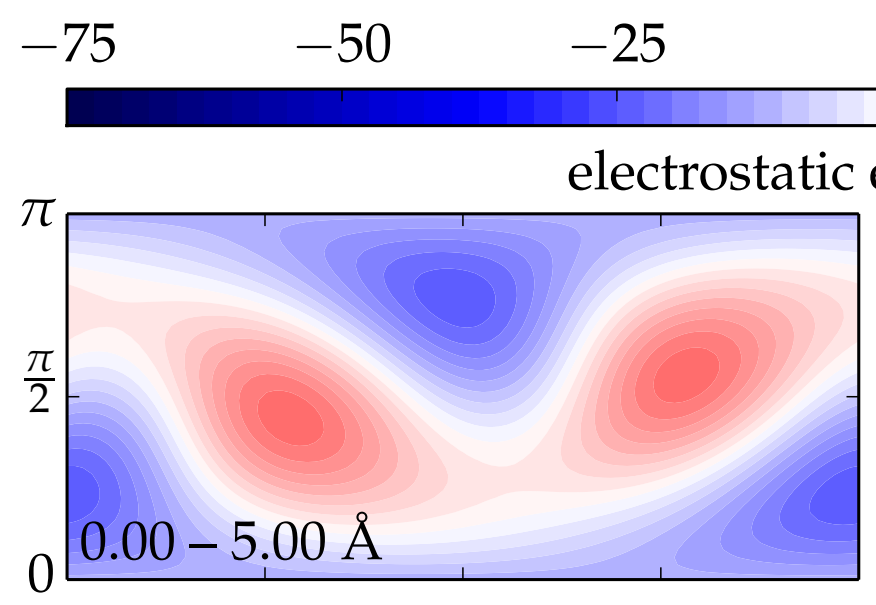

$\begin{array}{lr}0 & 25 \\ \text { energy }\left[\mathrm{cm}^{-1}\right]\end{array}$
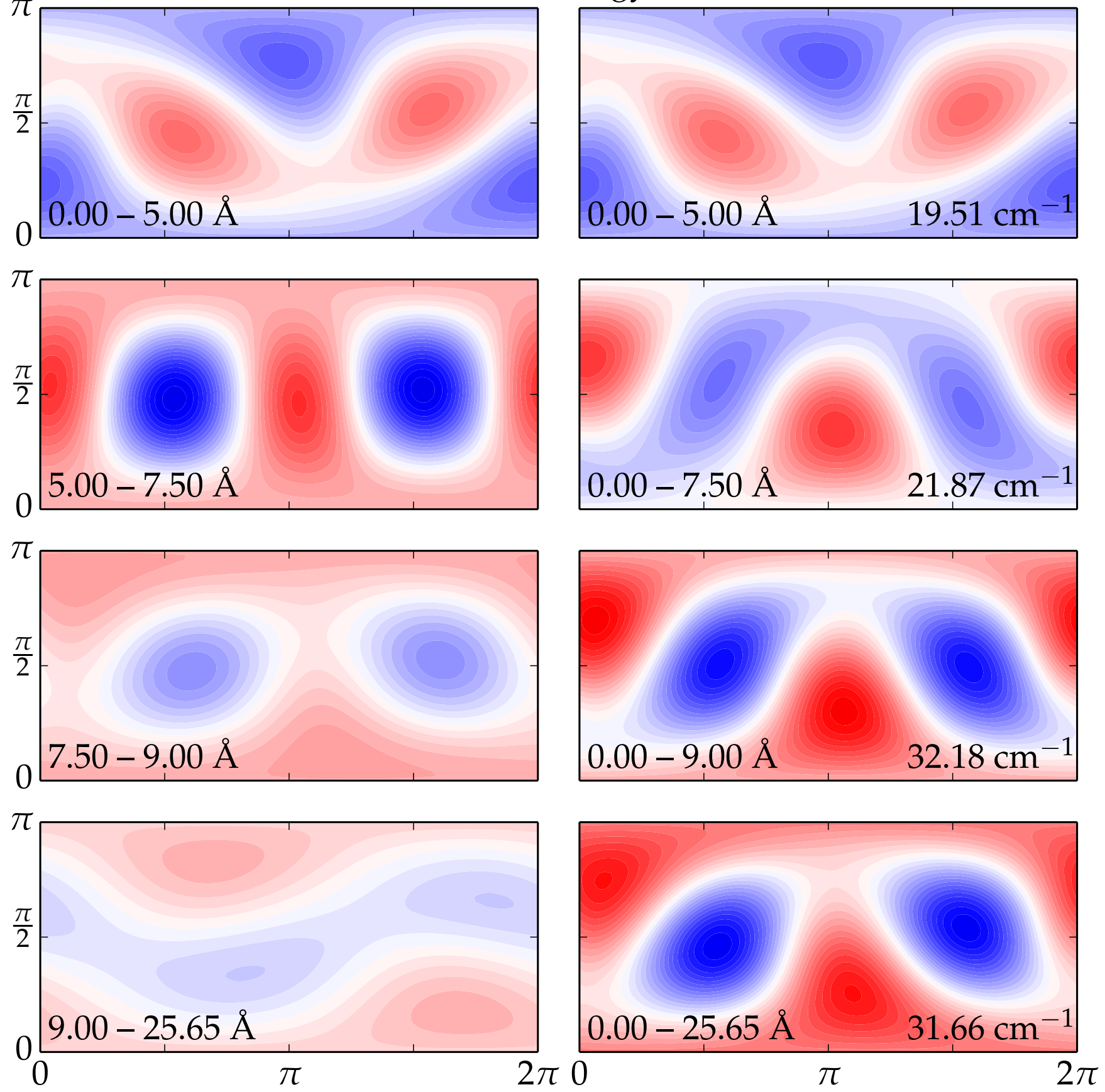

FIG. S1: The 2D electrostatic potential of $\mathrm{H}_{2}$ in a small cage, as a function of the polar angles $\theta$ and $\phi$ defining its orientation relative to the water framework. The c.m. of $\mathrm{H}_{2}$ is fixed at the center of the cage. The bottom left corner of each panel shows the range of distances $r$ from the cage center for which the water molecules contribute to the potential shown: $r=0.0-5.0$ $\AA$, the first solvation shell with 20 water molecules; $r=5.0-7.5 \AA$, the second solvation shell having 20 water molecules; $r=7.5-9.0 \AA$, the third solvation shell with 32 water molecules; $r=9.00-25.65 \AA, 1800$ water molecules. The panels in the left column show the separate contributions to the electrostatic potential due to the water molecules in each of the four shells. The panels in the right column show the cumulative electrostatic potentials arising from all water molecules within a sphere of a given radius; the corresponding $j=1$ rotational splittings calculated taking these water molecules into account appear in the bottom right corner of each panel. 


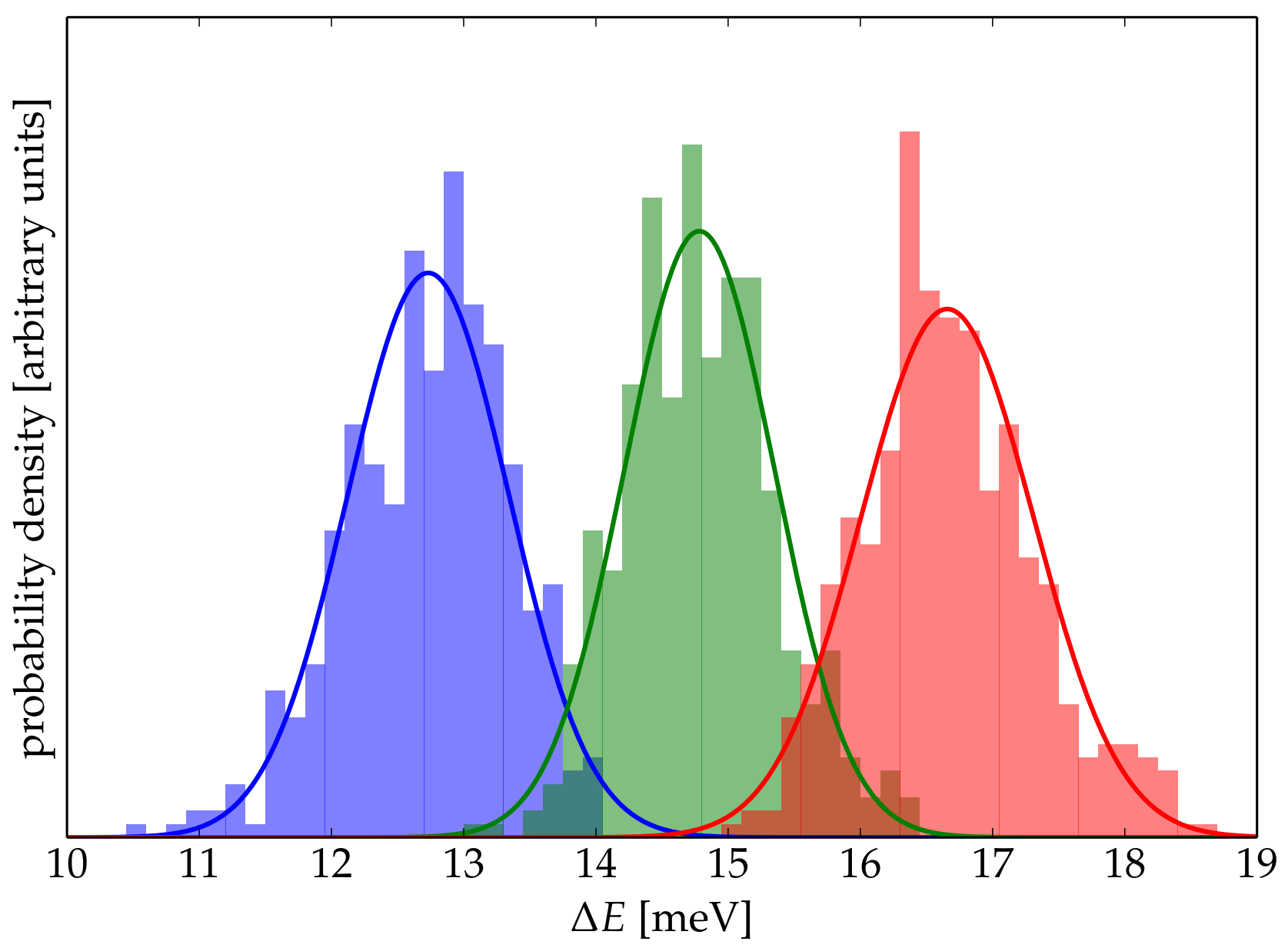

FIG. S2: Distribution of the three components of the $j=1$ rotational level calculated for 432 small cages in their respective condensed-phase environments ( $r=25.65 \AA$, 1872 water molecules). 
${ }^{1}$ M. Xu, Y. Elmatad, F. Sebastianelli, J. W. Moskowitz, and Z. Bačić. Hydrogen molecule in the small dodecahedral cage of a clathrate hydrate: Quantum five-dimensional calculations of the coupled translation-rotation eigenstates. J. Phys. Chem. $B, 110: 24806,2006$.

2 M. Xu, F. Sebastianelli, and Z. Bačić. Quantum dynamics of $\mathrm{H}_{2}, \mathrm{D}_{2}$, and HD in the small dodecahedral cage of clathrate hydrate: Evaluating $\mathrm{H}_{2}$-water nanocage interaction potentials by comparison of theory with inelastic neutron scattering experiments. J. Chem. Phys., 128:244715, 2008.

${ }^{3}$ M. Xu and Z. Bačić. Inelastic neutron scattering spectra of a hydrogen molecule in a nanocavity: Methodology for quantum calculations incorporating the coupled five-dimensional translation-rotation eigenstates. Phys. Rev. B, 84:195445, 2011.

${ }^{4} \mathrm{~S}$. Liu, Z. Bačić, J. W. Moskowitz, and K. E. Schmidt. Isomer dependence of HF vibrational frequency shift for Ar ${ }_{n} \mathrm{HF}$ $(n=4-14)$ van der Waals clusters: Quantum five-dimensional bound state calculations. J. Chem. Phys., 103:1829, 1995.

${ }_{5}$ M. Xu, F. Sebastianelli, and Z. Bačić. Coupled translation-rotation eigenstates of $\mathrm{H}_{2}, \mathrm{HD}$, and $\mathrm{D}_{2}$ in the large cage of structure II clathrate hydrate: Comparison with the small cage and rotational Raman spectroscopy. J. Phys. Chem. A, 113:7601, 2009.

${ }^{6}$ M. Xu, L. Ulivi, M. Celli, D. Colognesi, and Z. Bačić. Quantum calculation of inelastic neutron-scattering spectra of a hydrogen molecule inside a nanoscale cavity based on rigorous treatment of the coupled translation-rotation dynamics. Phys. Rev. B, 83:241403(R), 2011.

7 M. Xu, L. Ulivi, M. Celli, D. Colognesi, and Z. Bačić. Rigorous quantum treatment of inelastic neutron scattering spectra of a heteronuclear diatomic molecule in a nanocavity: HD in the small cage of structure II clathrate hydrate. Chem. Phys. Lett., 563:1, 2013.

${ }^{8}$ D. Colognesi, M. Celli, L. Ulivi, M. Xu, and Z. Bačić. Neutron scattering measurements and computation of the quantum dynamics of hydrogen molecules trapped in the small and large cages of clathrate hydrates. J. Phys. Chem. A, 117:7314, 2013.

9 D. Colognesi, A. Powers, M. Celli, M. Xu, Z. Bačić, and L. Ulivi. The HD molecule in small and medium cages of clathrate hydrates: Quantum dynamics studied by neutron scattering measurements and computation. J. Chem. Phys., 141:134501, 2014.

${ }^{10}$ M. Celli, A. Powers, D. Colognesi, M. Xu, Z. Bačić, and L. Ulivi. Experimental inelastic neutron scattering spectrum of hydrogen hexagonal clathrate-hydrate compared with rigorous quantum simulations. J. Chem. Phys., 139:164507, 2013.

11 S. Alavi, J. A. Ripmeester, and D. D. Klug. Molecular-dynamics study of structure II hydrogen clathrates. J. Chem. Phys., 123:024507, 2005.

12 H. J. C. Berendsen, J. R. Grigera, and T. P. Straatsma. The missing term in effective pair potentials. J. Phys. Chem., 91:6269, 1987.

13 Tom Darden, Darrin York, and Lee Pedersen. Particle mesh Ewald: An Nlog(N) method for Ewald sums in large systems. The Journal of Chemical Physics, 98(12):10089, 1993.

${ }^{14}$ Ulrich Essmann, Lalith Perera, Max L. Berkowitz, Tom Darden, Hsing Lee, and Lee G. Pedersen. A smooth particle mesh Ewald method. The Journal of Chemical Physics, 103(19):8577, 1995.

15 Sander Pronk, Szilárd Páll, Roland Schulz, Per Larsson, Pär Bjelkmar, Rossen Apostolov, Michael R Shirts, Jeremy C Smith, Peter M Kasson, David van der Spoel, Berk Hess, and Erik Lindahl. GROMACS 4.5: a high-throughput and highly parallel open source molecular simulation toolkit. Bioinformatics (Oxford, England), 29(7):845-54, April 2013. 